\title{
BiTeI Kristalinde Spin-Yörünge Yarılmasının Gerinim ile Değişimi
}

\author{
Sümeyra GÜLER-KILIÇ*, Çetin KILIÇ \\ Gebze Teknik Üniversitesi, Fizik Bölümü, Kocaeli
}

\begin{abstract}
Öz
BiTeI kristalinin elektronik bant yapısında, güçlü spin-yörünge etkileşmesi Rashba tipi spin ayrışmasına yol açmaktadır. Bu nedenle, dar bant aralıklı bir yariiletken olan BiTeI potansiyel bir spintronik malzeme olarak ilgi çekmektedir. Bu makalede, BiTel'deki Rashba tipi spin yarılmaların gerinim ile nasıl değiştiği incelenmiştir. Bu amaçla, yoğunluk fonksiyonel teorisi çerçevesinde kristal yapısı optimizasyonları ve bant yapısı hesaplamaları gerçekleştirilmiştir. Bu hesaplamaların sonuçları, BiTel'deki Rashba tipi spin yarılmasının gerinim ile kontrol edilebileceğini göstermektedir. Bu, BiTeI kristalinde gerinimin spin-yörünge etkileşimini arttırdığı (kristal sıkıştırıldığında) ya da azalttığı (kristal genleştiğinde) bulgusu ile açıklanmıştır.
\end{abstract}

Anahtar kelimeler: Spintronik yariiletkenler, BiTeI, Rashba tipi spin yarılması, Spin-yörünge etkileşimi, İlkprensip hesaplamalar.

\section{Variation of Spin-Orbit Splitting with Strain in BiTeI Crystal}

\begin{abstract}
In the electronic band structure of the BiTeI crystal, strong spin-orbit interaction leads to Rashba-type spin splitting. For this reason, BiTeI, a narrow band gap semiconductor, attracts attention as a potential spintronic material. In this article, it is examined how Rashba type spin splitting in BiTeI varies with strain. For this purpose, crystal structure optimizations and band structure calculations have been performed within the framework of density functional theory. The results of these calculations show that Rashba type splitting in BiTeI can be controlled with strain. This is explained by the finding that strain in the BiTeI crystal make the spin-orbit interaction increase (when the crystal compressed) or decrease (when the crystal dilated).
\end{abstract}

Keywords: Spintronic semiconductors, BiTeI, Rashba type spin splitting, Spin-orbit interaction, First-principles calculations.

\section{Giriş}

2011 yılında elektronik bant yapısında devasa Rashba tipi spin yarılmaları gözlemlendiğinden [1] beri dar bant aralıklı BiTeI yarıiletkeni, potansiyel bir spintronik malzeme olarak, büyük ilgi görmektedir. Rashba tipi yarılmalar yariiletken elektronlarının spin polarizasyonunun harici elektrik alanlarla kontrol edilmesine olanak sağladığı $[2,3]$ için BiTel'nin spintronik cihazlarda kullanımı cazip görülmektedir. BiTeI basınç altında topolojik faz geçişi göstermesi [4-10] ve termoelektrik özellikleri [11-13] ile de dikkat toplamaktadır.

Rashba etkisinin ilk olarak incelendiği sistem iki-boyutlu (2B) elektron gazıdır [14]. Bu etkinin tezahürü, güçlü spin-yörünge etkileşmesinin varlığına ve evirme simetrisinin yokluğuna bağlıdır. Ağır eleman içeren düşük-boyutlu bir yarıiletken için bu şartların sağlanması nispeten kolaydır. Bununla beraber, Rashba etkisinin belirgin bir şekilde ortaya çıktığı üç-boyutlu (3B) kristallerin sayısı son derece azdır. Çünkü 3B bir kristalde Rashba tipi yarılmaların oluşması istisnai ve nadirdir. BiTeI kristalinin yoğun ilgi çekmesinin arkasında bu vardır.

*Sorumlu yazar: sumeyra@gtu.edu.tr

Geliş Tarihi: 10.09.2018, Kabul Tarihi: 19.11.2018 
BiTeI yarıiletkeninin spin-ayrışmış Rashba bantlarına ait ölçüm [1,15-17] ve hesaplama [18-23] sonuçlarını rapor eden çok sayıda yayın çıkmaktadır. Katmanlı bir yapıya sahip olan BiTeI kristalini, ilk-prensip hesaplamaları gerçekleştirerek, incelediğimiz önceki bir yayınımızda [22], BiTeI bant yapısında oluşan Rashba tipi yarılmaların kökeni araştırılmıştır: BiTeI katmanlarının arasındaki bölgelerde içsel bir 2B elektron gazının oluştuğu açığa çıkarılmıştır. Bununla beraber, BiTeI katmanlarının kompozisyonunun birli olması ve bizmut, tellür ve iyot atomlarının elektronegatiflik değerlerinin uygun düşmesi sayesinde, zikredilen içsel 2B elektron gazının maruz kaldığ 1 asimetrik bir içsel elektrik alanın da var olduğu anlaşılmıştır. Bu asimetrik içsel elektrik alan, ağır eleman bizmutun varlığında spin-yörünge etkileşmesini belirleyerek, elektronik durumların spin eş-enerjiliğini ortadan kaldırmakta ve Rashba tipi yarılmaları netice vermektedir.

Rashba tipi spin yarılmaları, geleneksel olarak, e.g. [24], Rashba-Bychkov dispersiyon bağıntısı [14] kullanılarak karakterize edilmektedir. Aşağıda izah edildiği gibi, bu bağıntıya Rashba parametresi $\alpha$ ve elektronun etkin kütlesi $m^{*}$ girmektedir. Bu makalede BiTeI kristalindeki spin-yörünge yarılmasını betimleyen bu parametrelerin $\left(\alpha\right.$ ve $\left.m^{*}\right)$ gerinim ile nasıl değiştiği ele alınmaktadır. Bu amaçla BiTeI kristalinin birim hücre hacminin muhtelif değerleri için, yoğunluk fonksiyonel teorisi çerçevesinde, kristal yapısı optimizasyonları ve bant yapısı hesaplamaları gerçekleştirilmiş̧ir. Hacim değerleri kristalin hem genleşme hem de sıkışma durumlarını kapsayacak şekilde seçilmiştir. Böylece, Rashba parametrelerinin hacim (kristal genleştiğinde) ve basınç (kristal sıkıştırıldığında) ile gösterdiği değişim incelenmiştir.

\section{Materyal ve Metot}

BiTeI katmanlı trigonal bir yapıda kristalleşir; bu yapı, Şekil 1(a)'da gösterilmiştir. Bizmut, tellür ve iyot atomlarından oluşan ardışık birli katmanlar, bu katmanlara dik bir eksen boyunca dizilidirler; tellür ve iyot katmanlarının arasında van der Waals aralı̆̆ olarak isimlendirilen vakum bölgesi bulunmaktadır. BiTeI kristalinin uzay grubu, evirme simetrisini içermeyen, P3ml (No. 156) grubudur. $\mathrm{Bu}$ uzay grubuna ait Brillouin bölgesi Şekil 1(b)'de verilmiştir. Elektronik durumların momentum $(k)$ ekseni boyunca Rashba tipi spin ayrışmaları Brillouin bölgesinin H-A ve A-L çizgilerinde olmaktadır. $\mathrm{Bu}$ nedenle, aşağıda verilen bant yapısı grafikleri H-A ve A-L çizgileri üzerindeki $k$ değerleri kullanılarak çizilmiştir.

(a)

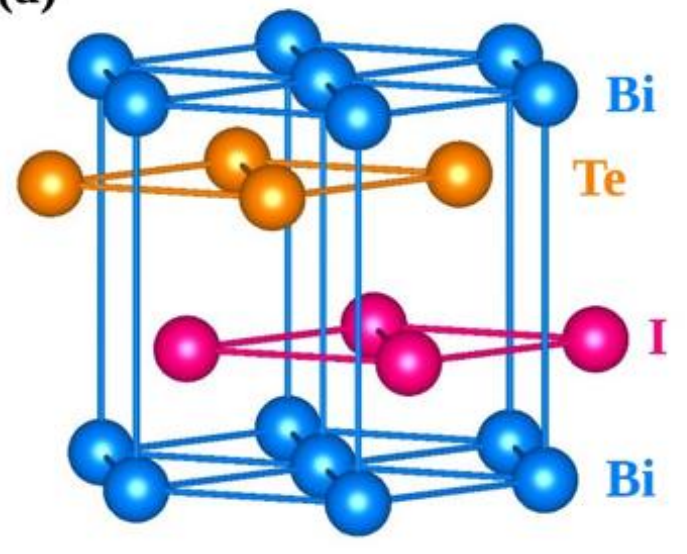

(b)

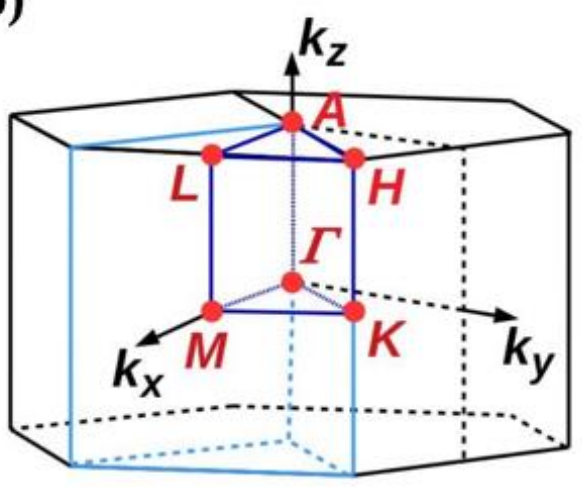

Şekil 1. BiTel kristalinin atomik yapısı (a), Brillouin bölgesi (b)

Kristal yapısı optimizasyonları ve bant yapısı hesaplamaları, yoğunluk fonksiyonel teorisi çerçevesinde, Vienna ab initio simülasyon paketi (VASP) [25] kullanılarak gerçekleştirilmiştir. Spinyörünge etkileşimi VASP kolineer-olmayan modda $[26,27]$ çalıştırılarak, hiçbir ayarlanabilir parametre kullanılmaksızın, hesaba katılmıştır. Değiş-tokuş-ilgileşim fonksiyoneli olarak Perdew-BurkeErnzerhof (PBE) fonksiyoneli [28] kullanılmıştır. Önceki bir yayınımızda [22] van der Waals dispersiyon etkileşmesinin hesaba katılmasının gerekli olduğu açığa çıkmıştır. Bu nedenle, PBE fonksiyoneline ilaveten yarı-ampirik bir dispersiyon potansiyeli [29] kullanılmıştır. Hesaplamalarda izdüşümsel ilaveli-dalga (PAW) yöntemi $[30,31]$ kullanılmış; tellür ve iyot için $5 s$ ve $5 p$ durumları ve 
bizmut için $6 s$ ve $6 p$ durumları değerlik durumları olarak değerlendirilmiştir. Elektronik durumlar, düzlem dalga baz setleri kullanılarak temsil edilmiştir; kinetik enerjinin kesim değeri 325 eV'dir.

Yukarıda belirtildiği gibi gerek deneysel gerekse ilk-prensip hesaplama yöntemleri ile elde edilen, spin-ayrışmış Rashba bantlarının incelenmesinde,

$$
\epsilon_{ \pm}(k)=\frac{\hbar^{2}}{2 m^{*}} k^{2} \pm \alpha k
$$

ifadesiyle verilen Rashba-Bychkov dispersiyon bağıntıs1 [14] yarar sağlamaktadır. Burada, $\alpha$ ve $m^{*}$, sıra ile, Rashba parametresi ve etkin kütleyi göstermektedir. Spin yarılma enerjisi $k$ 'da lineerdir ve $\alpha$ ile doğru orantılıdır: $\Delta \epsilon(k)=\epsilon_{+}(k)-\epsilon_{-}(k)=2 \alpha k$. Bant enerjisi $\epsilon_{ \pm}(k)$ 'nin minimum değeri aldığı $k$ değeri momentum ofseti olarak adlandırılır ve

$$
k_{0}=\frac{m^{*} \alpha}{\hbar^{2}}
$$

ifadesiyle verilir. Spin yarılmasının enerjik bir ölçütü olarak,

$$
E_{R}=\frac{\alpha k_{0}}{2}
$$

ifadesiyle verilen Rashba enerjisi kullanılmaktadır. Aşağıda Şekil 2'de gösterildiği gibi, bant yapısı grafiklerinden, $k_{0}$ ve $E_{R}$ 'nin değerleri doğrudan elde edilmektedir. Bu değerler Eşitlik (2) ve (3)'e yerleştirilerek ve bu eşitlikler $\alpha$ ve $m^{*}$ için çözülerek, Rashba parametresi ve etkin kütle elde edilmektedir.

BiTeI'nin birim hücre hacmi $V$ 'nin muhtelif değerleri için kristal yapısı optimizasyonları gerçekleştirilerek, önceki bir yayınımızda [22] detaylı olarak anlatıldığı gibi, BiTeI kristalinin durum denklemi türetilmiştir. Böylece basınç-hacim $P-V$ denklemi elde edilmiştir. Aşağıda $V$ 'nin denge değeri, yani $P=0$ için aldığı değer, $V_{0}$ olarak gösterilmiştir. Gerinim ölçütü olarak, hacim oranı $V / V_{0}$ kullanılmıştır. Bu oranın birden büyük ve küçük olması, sıra ile, kristalin genleşmesi ve sıkıştırılması anlamına gelmektedir. Her hacim değeri için, bant yapısı hesaplamaları yapılarak, Rashba parametrelerinin muhtelif hacim değerlerine karşılık gelen değerleri elde edilmiştir. Böylece, Rashba parametresi ve etkin kütlenin hacim $V$ (kristal genleştiğinde) ve basınç $P$ (kristal sıkıştırıldığında) ile nasıl değiştiği belirlenmiştir.

\section{Bulgular ve Tartışma}

BiTeI kristalinin muhtelif hacim $V$ değerleri için elde edilen elektronik bant yapısı grafikleri Şekil 2'de verilmiştir; en düşük (yüksek) enerjiye sahip olan iki iletim (değerlik) bandı çizilmiştir. Hem iletim hem de değerlik bantlarının momentum ekseninde birbirinden ayrışması spin-yörünge etkileşmesinden kaynaklanmaktadır. Hacim oranı $V / V_{0}$ değiştikçe hem bant aralık enerjisi hem de bantların şekli (dispersiyonu) değişim göstermektedir. Bant aralığı enerjisinin basınç ile gösterdiği değişim önceki bir yayınımızda [10] detaylı olarak ele alınmıştır. Özetle, basıncın kritik bir değerine $\left(P_{c}\right)$ kadar bant aralığı küçülmekte; $P=P_{c}$ olduğunda bant aralığı tamamen kapanmakta ve BiTeI yarı metale dönüşmektedir. Basınç artarken kritik basınç $P_{c}$ değeri geçildiğinde ise, bant aralığı tekrar açılmaktadır. Buna, kristal genleşirken $\left(V / V_{0}>1\right)$ bant aralığının artma eğiliminde olduğu ilave edilmelidir. 

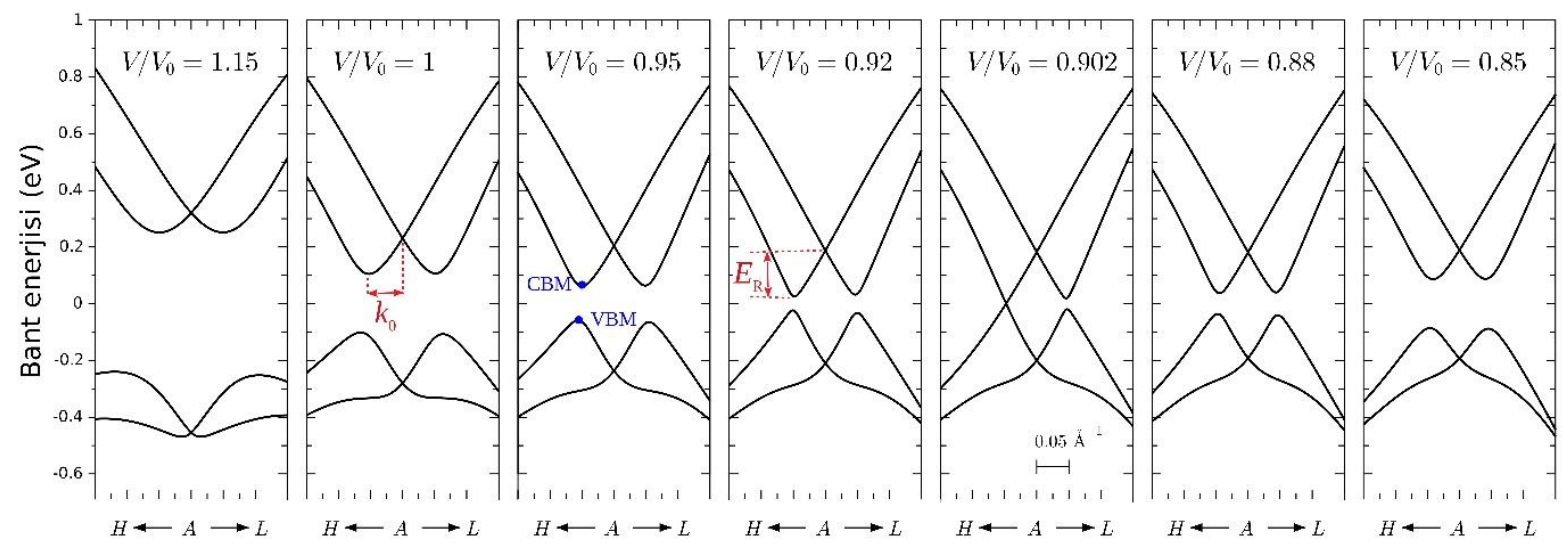

Şekil 2. BiTeI kristalinin, hacim oranı $V / V_{0}$ 'nın muhtelif değerleri için, elektronik bant yapısı grafikleri; sadece dört bant (en düşük enerjiye sahip iki iletim bandı ve en yüksek enerjiye sahip iki değerlik bandı) çizilmiştir. Değerlik bandı maksimumu ve iletim bandı minimumu, sıra ile VBM ve CBM olarak işaretlenmiş; Rashba enerjisi $\left(E_{R}\right)$ ve momentum ofseti $\left(k_{0}\right)$ gösterilmiştir.
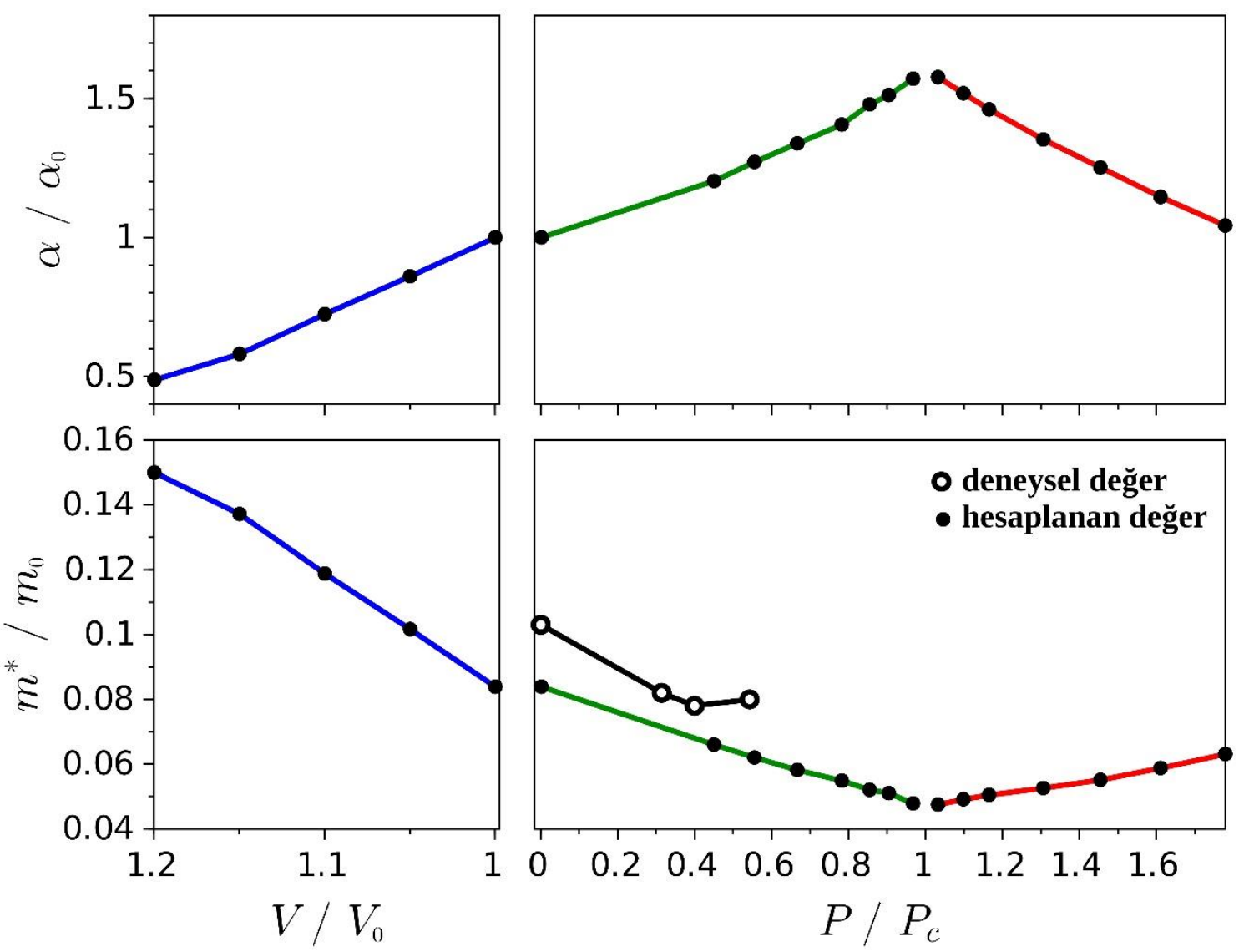

Şekil 3. BiTeI kristali için Rashba parametresi $\alpha$ ve etkin kütle $m^{*}$ 'nin hacim oranı $V / V_{0}$ ve basınç oranı $P / P_{c}$ ile değişimi; $m_{0}$ serbest elektron kütlesini, $\alpha_{0}$ Rashba parametresinin $V=V_{0}$ için değerini göstermektedir. Deneysel değerleri şu kaynaklardan alınmıştır: [1], [32].

Şekil 2'de hacim oranı $V / V_{0}$ 'nın muhtelif değerleri için bant dispersiyonun farklı olması, momentum ofseti, Rashba enerjisi ve etkin kütlenin gerinim ile değiştiğinin bir göstergesidir. Bu, BiTeI yariiletkeninin elektronik yapısına spin-yörünge etkileşmesinden gelen katkının gerinim ile kontrol edilebileceğini göstermektedir. Rashba Hamiltoniyeninde spin-yörünge etkileşiminin katkısını $\alpha$ 
parametresinin değeri belirlemektedir. Bu nedenle, Rashba parametresi $\alpha$ 'nın gerinim ile nasıl değiştiği incelenmiştir. Şekil 3'ün üst panellerinde $\alpha / \alpha_{0}$ oranı, hacim oranı $V / V_{0}$ 'ye (sol panel) ve basinç oranı $P / P_{c}$ 'ye (sağ panel) göre çizilmiştir; $\alpha_{0}$ Rashba parametresinin $V=V_{0}$ için aldığ değeri göstermektedir. Şekil 3'ün alt panellerinde ise $\mathrm{m}^{*} / \mathrm{m}_{0}$ oranı aynı gerinim ölçütlerine göre çizilmiştir; $m_{0}$ serbest elektron kütlesini göstermektedir. Şekil 3'de mavi, yeşil ve kırmızı çizgilerle birleştirilen noktalar üç farklı duruma tekabül etmektedir: (i) Kristal genleşirken, $\alpha / \alpha_{0}\left(m^{*} / m_{0}\right)$ oranı $V / V_{0}$ ile yaklaşık olarak doğru orantılı bir şekilde azalmaktadır (artmaktadır). Bu durumda, BiTeI katmanları arasındaki van der Waals aralığı büyüdüğü için spin-yörünge etkileşimi zayıflamakta ve yariiletken elektronları "ağır"laşmaktadır. (ii) Kristal sıkıştırılırken, basınç kritik değerden küçük ise, $\alpha / \alpha_{0}$ $\left(m^{*} / m_{0}\right)$ oranı $P / P_{c}$ ile yaklaşık olarak doğru orantılı bir şekilde artmaktadır (azalmaktadır). Bu durumda, spin-yörünge etkileşimi güçlenmekte ve yariletken elektronları "hafif"lemektedir. Hesaplanan $m^{*} / m_{0}$ değerlerinin deneysel [1,32] değerlerle oldukça uyumlu olduğu belirtilmelidir. (iii) Kristal sıkıştırılırken, basınç kritik değerinden büyük ise, $\alpha / \alpha_{0}\left(m^{*} / m_{0}\right)$ oranı $P / P_{c}$ ile yaklaşık olarak doğru orantılı bir şekilde azalmaktadır (artmaktadır). Bu bulgular, BiTeI yarilletkeninde spinyörünge etkileşim şiddetinin gerinim ile kontrol edilebileceğini göstermektedir.

\section{Sonuç ve Öneriler}

$\mathrm{Bu}$ makalede, yoğunluk fonksiyonel teorisi çerçevesinde ilk-prensip hesaplamaları gerçekleştirilerek BiTeI yariiletkeninde elektronik bant dispersiyonunun ve Rashba tipi spin yarılmasının gerinim ile nasıl değiştiği araştırılmıştır. BiTeI kristalinde gerinimin spin-yörünge etkileşmesinin şiddetini değiştirdiği bulunmuştur: Kristal sıkıştırılırken spin-yörünge etkileşimi artmakta, kristal genleşirken ise azalmaktadır. Bu, BiTeI yarıiletkenindeki Rashba tipi spin yarılmasının gerinim ile gösterdiğgi değişimi açıklamaktadır. $\mathrm{Bu}$ bulgular, BiTeI yariiletkeninin spintronik özelliklerinin gerinim ile kontrol edilebileceğini göstermektedir.

\section{Teșekkür}

Nümerik hesaplamalar TÜBİTAK ULAKBİM TRUBA kaynaklarında gerçekleştirilmiştir.

\section{Kaynaklar}

[1] Ishizaka K., Bahramy M.S., Murakawa H., Sakano M., Shimojima T., Sonobe T., Koizumi K., Shin S., Miyahara H., Kimura A., Miyamoto K., Okuda T., Namatame H., Taniguchi M., Arita R., Nagaosa N., Kobayashi K., Murakami Y., Kumai R., Kaneko Y., Onose Y., Tokura Y. 2011. Giant Rashba-type Spin Splitting in Bulk BiTeI, Nature Mater., 10: 521-526.

[2] Hoffmann A., Bader S.D. 2015. Opportunities at the Frontiers of Spintronics, Phys. Rev. Applied, 4 (047001): 1-18.

[3] Silsbee R. H. 2004. Spin-Orbit Induced Coupling of Charge Current and Spin Polarization, J. Phys.: Condens. Matter, 16: R179-R207.

[4] Bahramy M.S., Yang B.J., Arita R., Nagaosa N. 2012. Emergence of Non-Centrosymmetric Topological Insulating Phase in BiTeI under Pressure, Nature Commun., 3 (679): 1-7.

[5] Xi X., Ma C., Liu Z., Chen Z., Ku W., Berger H., Martin C., Tanner D. B., Carr G. L. 2013. Signatures of a Pressure-Induced Topological Quantum Phase Transition in BiTeI, Phys. Rev. Lett., 11 (155701): 1-5.

[6] Tran M.K., Levallois J., Lerch P., Teyssier J., Kuzmenko A.B., Autès G., Yazyev O.V., Ubaldini A., Giannini E., van der Marel D., Akrap A. 2014. Infrared- and Raman-Spectroscopy Measurements of a Transition in the Crystal Structure and a Closing of the Energy Gap of BiTeI under Pressure, Phys. Rev. Lett., 112 (047402): 1-5.

[7] Liu J., Vanderbilt D. 2014. Weyl Semimetals from Noncentrosymmetric Topological Insulators, Phys. Rev. B, 90 (155316): 1-10. 
[8] Ideue T., Checkelsky J.G., Bahramy M.S., Murakawa H., Kaneko Y., Nagaosa N., Tokura Y. 2014. Pressure Variation of Rashba Spin Splitting Toward Topological Transition in the Polar Semiconductor BiTeI, Phys. Rev. B, 90 (161107): 1-5.

[9] Park J., Jin K.-H., Jo Y. J., Choi E. S., Kang W., Kampert E., Rhyee J.-S., Jhi S.-H., Kim J. S. 2015. Quantum Oscillation Signatures of Pressure-induced Topological Phase Transition in BiTeI, Sci. Rep., 5 (15973): 1-8.

[10] Güler-Kılıç S., Kılıç Ç. 2016. Pressure Dependence of the Band-Gap Energy in BiTeI, Phys. Rev. B, 94 (165203): 1-7.

[11] Kulbachinskii V.A., Kytin V.G., Lavrukhina Z.V., Kuznetsov A.N., Shevelkov A.V. 2010. Galvanomagnetic and Thermoelectric Properties of BiTeBr and BiTeI Single Crystals and their Electronic Structure, Semiconductors, 44 (12): 1548-1553.

[12] Wu L., Yang J., Chi M., Wang S., Wei P., Zhang W., Chen L., Yang J. 2015. Enhanced Thermoelectric Performance in $\mathrm{Cu}$-Intercalated BiTeI by Compensation Weakening Induced Mobility Improvement, Sci. Rep., 5 (14319): 1-12.

[13] Wu L., Yang J., Zhang T., Wang S., Wei P., Zhang W., Chen L., Yang J. 2016. Enhanced Thermoelectric Performance in the Rashba Semiconductor BiTeI Through Band Gap Engineering, J. Phys.: Condens. Matter, 28 (085801): 1-7.

[14] Bychkov Y.A., Rashba E.I. 1984. Properties of a 2D Electron Gas with Lifted Spectral Degeneracy, JETP Lett., 39 (2): 78-81.

[15] Landolt G., Eremeev S.V., Koroteev Y.M., Slomski B., Muff S., Neupert T., Kobayashi M., Strocov V.N., Schmitt T., Aliev Z.S., Babanly M.B., Amiraslanov I.R., Chulkov E.V., Osterwalder J., Dil J.H. 2012. Disentanglement of Surface and Bulk Rashba Spin Splittings in Noncentrosymmetric BiTeI, Phys. Rev. Lett., 109 (116403): 1-5.

[16] Sakano M., Miyawaki J., Chainani A., Takata Y., Sonobe T., Shimojima T., Oura M., Shin S., Bahramy M.S., Arita R., Nagaosa N., Murakawa H., Kaneko Y., Tokura Y., Ishizaka K. 2012. ThreeDimensional Bulk Band Dispersion in Polar BiTeI with Giant Rashba-Type Spin Splitting, Phys. Rev. B, 86 (085204): 1-5.

[17] Kanou M., Sasagawa T. 2013. Crystal Growth and Electronic Properties of a 3D Rashba Material, BiTeI, with Adjusted Carrier Concentrations, J. Phys.: Condensed Matter, 25 (135801): 1-6.

[18] Bahramy M.S., Arita R., Nagaosa N. 2011. Origin of Giant Bulk Rashba Splitting: Application to BiTeI, Phys. Rev. B, 84 (041202): 1-4.

[19] Fu H. 2013. Tunability of Giant Rashba Spin Splitting in BiTeI, Phys. Rev. B, 87 (075139): 1-6.

[20] Rusinov I.P., Nechaev I.A., Eremeev S.V., Friedrich C., Blügel S., Chulkov E.V. 2013. Many-Body Effects on the Rashba-Type Spin Splitting in Bulk Bismuth Tellurohalides, Phys. Rev. B, 87 (205103): 1-5.

[21] Zhu Z., Cheng Y., Schwingenschlögl U. 2013. Orbital-Dependent Rashba Coupling in Bulk BiTeCl and BiTeI, New J. Phys., 15 (023010): 1-8.

[22] Güler-Kılıç S., Kılıç Ç. 2015. Crystal and Electronic Structure of BiTeI, AuTeI, and PdTeI Compounds: A Dispersion-Corrected Density-Functional Study, Phys. Rev. B, 91 (245204): 1-12.

[23] Schwalbe S., Wirnata R., Starke R., Schober G.A.H., Kortus J. 2016. Ab Initio Electronic Structure and Optical Conductivity of Bismuth Tellurohalides, Phys. Rev. B, 94 (205130): 1-7.

[24] Ast C.R., Pacilé D., Moreschini L., Falub M.C., Papagno M., Kern K., Grioni M., Henk J., Ernst A., Ostanin S., Bruno P. 2007. Giant Spin Splitting Through Surface Alloying, Phys. Rev. Lett., 98 (186807): 1-5.

[25] Kresse G., Furthmüller J. 1996. Efficient Iterative Schemes for Ab Initio Total-Energy Calculations Using a Plane-Wave Basis Set, Phys. Rev. B, 54 (16): 11169-11186.

[26] Hobbs D., Kresse G., Hafner J. 2000. Fully Unconstrained Noncollinear Magnetism within the Projector Augmented-Wave Method, Phys. Rev. B, 62 (17): 11556-11570.

[27] Steiner S., Khmelevskyi S., Marsmann M., Kresse G. 2016. Calculation of the Magnetic Anisotropy with Projected-Augmented-Wave Methodology and the Case Study of Disordered $\mathrm{Fe}_{1-\mathrm{x}} \mathrm{Co}_{\mathrm{x}}$ Alloys, Phys. Rev. B, 93 (224425): 1-6.

[28] Perdew J.P., Burke K., Ernzerhof M. 1996. Generalized Gradient Approximation Made Simple, Phys. Rev. Lett., 77 (18): 3865-3868.

[29] Grimme S. 2006. Semiempirical GGA-Type Density Functional Constructed with a Long-Range Dispersion Correction, J. Comput. Chem., 27 (15): 1787-1799. 
[30] Blöchl P.E. 1994. Projector Augmented-Wave Method, Phys. Rev. B, 50 (24): 17953-17979.

[31] Kresse G., Joubert J. 1999. From Ultrasoft Pseudopotentials to the Projector Augmented-Wave Method, Phys. Rev. B, 59 (3): 1758-1775.

[32] VanGennep D., Maiti S., Graf D., Tozer S.W., Martin C., Berger H., Maslov D.L., Hamlin J.J. 2014. Pressure Tuning the Fermi Level Through the Dirac Point of Giant Rashba Semiconductor BiTeI, J. Phys.: Condens. Matter, 26 (342202): 1-7. 